\title{
Rectal Ulcer, CTCAE
}

National Cancer Institute

\section{Source}

National Cancer Institute. Rectal Ulcer, CT CAE. NCI Thesaurus. Code C57867.

A disorder characterized by a circumscribed, erosive lesion on the mucosal surface of the rectum. 